\title{
Mini-EUSO data processing and quasi-real time analysis
}

\author{
Lech Wiktor Piotrowski* \\ RIKEN, Wako, Japan \\ E-mail: lech.piotrowski@riken.jp
}

\section{A. Belov}

Lomonosov Moscow State University, Skobeltsyn Institute of Nuclear Physics (SINP MSU), Moscow, Russia

Lomonosov Moscow State University, Faculty of Physics, Moscow, Russia

\author{
F. Capel \\ KTH Royal Institute of Technology, Stockholm, Sweden
}

\section{for the JEM-EUSO collaboration}

\begin{abstract}
Mini-EUSO is a telescope that will observe ultra-violet night-time light emission of Earth. It will watch the atmosphere through a UV transparent window of the International Space Station. The main data stream is composed of regularly sampled exposures integrated on three different time scales: microseconds, milliseconds and seconds. This, accompanied by visible and near infra red images will be sent to Earth on hard drives for analysis. We present here the general dataprocessing scheme for the mission, including the data format and the tools for quick view and first analysis of the collected data.
\end{abstract}

35th International Cosmic Ray Conference - ICRC2017

10-20 July, 2017

Bexco, Busan, Korea

${ }^{*}$ Speaker. 


\section{Introduction}

Cosmic rays - high-energy particles of extra-terrestrial origin - have proven to be important area of science since their discovery at the beginning of $20^{\text {th }}$ century. Further research has extended our knowledge about this phenomenon significantly, revealing most of the energy spectrum, composition and sources of this radiation. However, the extremely high part of the energy spectrum remains mysterious. We are still not sure if there is and what would be the cut-off energy and what in the Universe can accelerate particles to such huge velocities.

The main reason for those questions remaining unanswered is the very low flux of the most energetic cosmic rays reaching Earth. If one considers protons, nuclei and electrons, the rate for $10^{9} \mathrm{eV}$ particles is $\frac{10000}{\mathrm{~m}^{2} \cdot \mathrm{s}}$, but above $10^{20} \mathrm{eV}$ it drops to $\frac{1}{\mathrm{~km}^{2} \cdot 100 \mathrm{yr}}$, which makes the study in the highest energies regime extremely difficult. To obtain a statistically significant result in an average experiment lifespan, one has to monitor at least thousands square kilometres of ground. For the UV telescopes observing fluorescence light from the Extensive Air Showers caused by the primary cosmic ray, the volume of observed atmosphere is limited by their field of view and the distance to atmospheric surface, as well as several other factors. The coverage of a telescope could be significantly increased with increase of the distance to the atmospheric surface. Introducing observations of shower-induced UV light from orbit is the main idea behind the JEM-EUSO mission [1].

Realising this innovative concept requires introducing several preceding missions in the EUSO framework. One of them is Mini-EUSO, a space-borne UV telescope that will observe Earth's atmosphere from within the International Space Station (ISS) through a UV transparent window. The size constraints of the telescope lenses make Ultra High Energy Cosmic Rays (UHECR) detection a low priority, however it will be the first experiment to create a night-time UV map of the atmosphere. In addition, it will observe atmospheric phenomena such as TLEs and lightnings, meteors and will have strangelets detection capabilities.

Mini-EUSO [2] is approved as a joint project by the Italian (ASI) and Russian (Roscosmos) space agencies and is included in the long-term program of space experiments on the Russian segment of the ISS under the name "UV-Atmosphere". The integration of the instrument is currently at an advanced stage in order to be compliant with a launch opportunity in late 2017 to early 2018.

\section{The Mini-EUSO telescope}

The light will enter the Mini-EUSO telescope through a UV transparent window on the Russian ISS Zvezda module. It will be focused with 2 Fresnel lenses, each with diameter of $25 \mathrm{~cm}$, onto a single photon counter - the PhotoDetector Module (PDM). Its active surface is composed of 36 Multianode Photomultipliers (MAPMT), giving in total 2304 pixels (fig. 1). Four MAPMTs form an Elementary Cell (EC) unit, each with a dedicated Cockcroft-Walton based High Voltage Power Supply. The working voltage of the phototubes is $1100 \mathrm{~V}$, and is automatically reduced in case of too bright light on an EC-Unit.

The field of view of the instrument is $44^{\circ} \times 44^{\circ}$, with $\sim 0.9^{\circ}$ angular resolution. Given the average orbit of the ISS, this translates to $\sim 6 \mathrm{~km}$ spatial resolution on the ground level. 


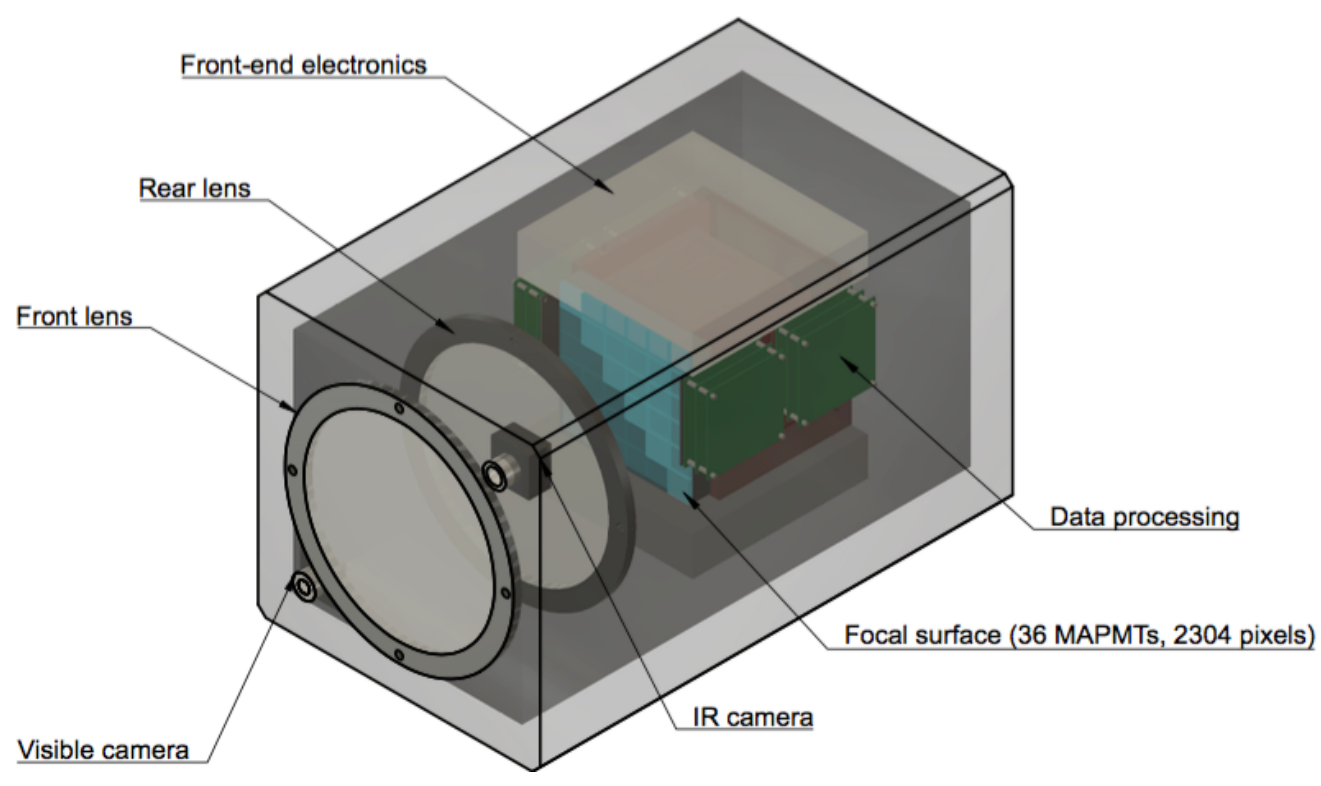

Figure 1: Schematic view of the Mini-EUSO instrument, showing two Fresnel lenses that focus light on the 2304 channel focal surface read out by front-end electronics. In addition, visual and near infra-red cameras, as well as data processing unit are visible.

\subsection{Data acquisition}

Each PMT is read out by one SPACIROC3 ASIC, which are distributed onto 6 EC-ASIC boards [3], integrating the counts into $2.5 \mu$ s frames - Gate Time Units (GTUs). Counts from all the boards are passed to a Xilinx Zynq XC7Z030 [4] board, which performs the triggering and sends selected events to a PCIe/104 form factor CPU. The data gathered by the CPU also contains images from near infra-red and visible cameras, as well as information from silicon photomultipliers, photodiode and temperature sensors, that are read out by the Housekeeping (HK) subsystem. Data is stored on the internal Solid State Drive (SSD) units.

It is not possible to download all of the data to Earth due to complicated procedure involving the data transfer. Therefore only small control packets and most interesting events will be send over the network, and the rest will be periodically carried down on SSDs.

The main part of data acquisition will be performed during the ISS's night, after confirmation that the dark period has started based on the information from photodiodes. PDM data will be stored every $5.24 \mathrm{~s}$, containing triggered [5] and untriggered packets with three sampling rates, as described in sec. 3. Data from VIS and NIR cameras will be taken in similar manner: when triggered by Mini-EUSO trigger and, additionally, every $5.24 \mathrm{~s}$, together with housekeeping, thermistor and silicon photomultipliers information. The data output rate in this mode is estimated to be $\sim 2 \mathrm{~TB} /$ month, assuming an $25 \%$ duty cycle.

During the daytime the CPU is utilised to produce a selection of the most interesting events that are downloaded to the ground along with basic diagnostic information and ancillary cameras images. The data budget foreseen for such purposes is of the order of $200 \mathrm{MB} / \mathrm{month}$. Mini-EUSO operations are described in more detail in [6]. 


\section{Data format}

\subsection{Raw data}

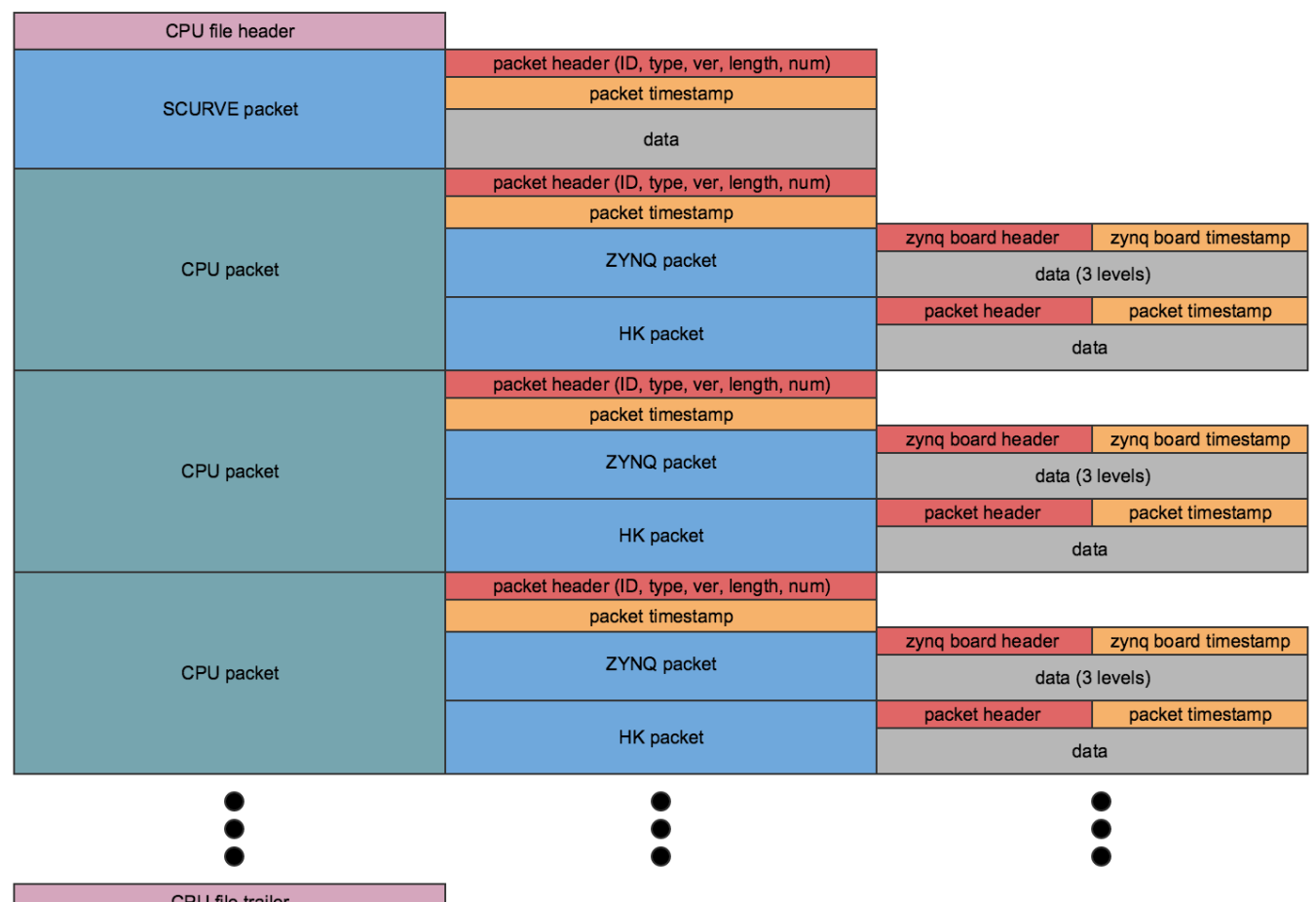

Figure 2: A scheme of packet organization in Mini-EUSO experiment. Left column shows a scheme of the file containing the whole night data. Consecutive columns show the contents of packets in the previous column.

The data gathering is made with encapsulated packets. The amount of data contained in a packet increases with the level of processing (fig. 2). A single packet of information contains counts for all photon counting channels (pixels) read from the EC-ASIC boards, integrated on three time scales (L1, L2, L3, fig. 3):

L1 128 frames, $2.5 \mu$ s each, stored when corresponding trigger is issued by the Zynq board. Each pixel is written as a single unsigned byte. These packets are dedicated for registering $\mu \mathrm{s}$ scale phenomena, such as Extensive Air Showers from cosmic rays.

L2 128 frames, $320 \mu$ s each (sum of $128 \times 2.5 \mu$ s frames), stored when corresponding trigger is issued by the Zynq board. Each pixel value is stored in two bytes. These packets are dedicated for registering phenomena such as Transient Luminous Events and lightnings in the upper atmosphere.

L3 128 frames, $40.96 \mathrm{~ms}$ each (sum of $128 \times 320 \mu$ s frames), stored continuously every $5.24 \mathrm{~s}$. Each pixel is stored in four bytes. These packets are dedicated for registering UV background map of Earth as a "movie", and are also suitable for study of slower moving objects, such as meteors, strangelets or space debris. 
Summing up, the 5.24 s packet contains just over $2 \mathrm{MB}$ of data written in dedicated, encapsulated $\mathrm{C}$ structures. Pointers to the structures of the lower level units are stored in the higher level unit structure, which adds its own data, to form a final file containing all the packets stored during the whole night (about 40 minutes for ISS). In addition, at the beginning of each night a special diagnostic data is stored - so called S-Curves - which are the count data for each pixel, but collected for a set of specific single-photon recognition thresholds. This enables monitoring of changes in the gain of each channel and adjusting the previously mentioned threshold.

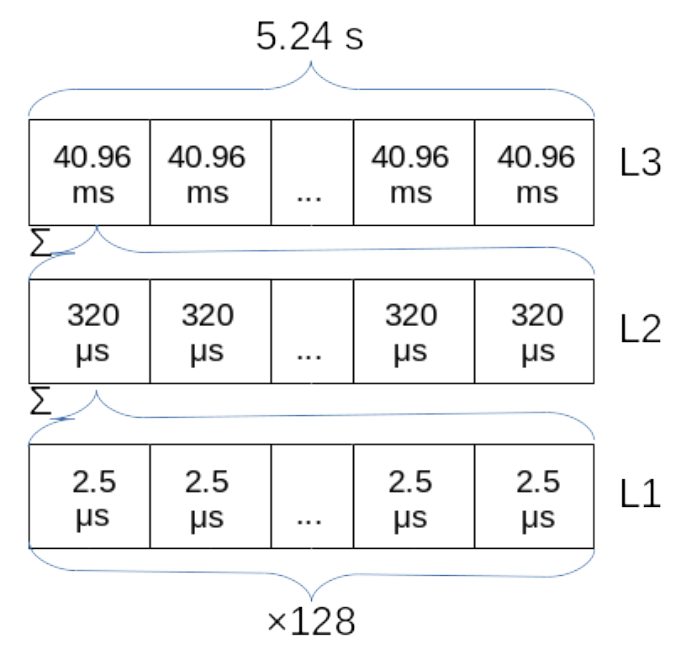

Figure 3: Scheme of the UV photon counts data structure, showing three time-scales of time integration, dedicated to different phenomena: L1 to UHECR-like events, L2 to atmospheric discharges and L3 to UV background mapping, meteors, strangelets, etc.

The data for ancillary cameras and silicon photomultipliers will be stored in their own formats.

\subsection{Processed data}

Sequential storing of binary packets coming directly from subsystems is very efficient in terms of writing speed, memory use and bandwidth conservation. However, it is very inefficient when accessing random parts of data, a basic task in data analysis. Moreover, in case of any change in the format or experiment design, different data sets become incompatible and maintaining access to them becomes a very complicated task. Therefore for data sharing and analysis purposes a different format has to be introduced. The requirements are:

1. fast access and searching

2. ease of use

3. ease of sharing and distribution

4. popularity in the scientific community that will use the data

5. ability to handle very big data sets 
While requirements 1,2 and 3 can be fulfilled by several data formats, it seems that in the UHECR community the requirements 4 and 5 are fulfilled only by Cern ROOT package [7] TTree format ${ }^{1}$. Therefore the TTrees were chosen as the basic format for data sharing and analysis and are used in other experiments within the JEM-EUSO collaboration.

The program for conversion from hardware format to TTrees simply analyses all the packets in the file sequentially converting them and assigning to separate TTrees. The Mini-EUSO ROOT file currently includes following types of TTrees:

texp Information about the experiment and specific run

tcal Calibration (S-Curve) data

tevent $\mathrm{L} 1$ timescale photon counts data

tevent_1st_integral L2 timescale photon counts

tevent_2nd_integral L3 timescale photon counts

The first three TTrees are common for all existing JEM-EUSO experiments, while the tress for L2 and L3 timescales are specific to Mini-EUSO at the moment, however will be introduced to some of the future missions.

The speed of conversion to the TTrees, as well as efficiency of the format allows for developing a real-time monitoring of the system. The object oriented design, C++ syntax and Python bindings makes development of an analysis software convenient. The experiment's TTrees have a versatile, scalable design, easily accommodating different experimental designs. These features make the format transparent - the data can be analysed in exactly the same way regardless of whether it was gathered in a real experiment or generated by a Monte-Carlo simulator.

The proprietary data formats of the ancillary cameras are converted to PNG files for the ease of viewing.

\section{Quick-view and data analysis}

The download of preselected packets allows for semi-real time data analysis. Data stored in TTrees can be read and visualised by quick-view software specially designed for this task - "Etos" (Euso TO Screen) - and automatically updated in real time, if requested. The main requirements during development of this program were convenience of use, simplicity of distribution and installation and simplicity of adding new features.

The software has been written in Python with ROOT bindings - PyROOT. This allows for rapid development and easy use of an extensive library of Python scientific packages, such as NumPy or SciPy along the built-in ROOT functions. This is especially important for a program with basic data analysis capabilities - many of user requirements are not known at the time of designing the software and often need to be quickly implemented and used during hardware development or data acquisition sessions. In addition to different features required by different developers of the

\footnotetext{
${ }^{1}$ Actually, the authors could not find any other format that can be easily shared, is fast to access and works well with very big data sets.
} 


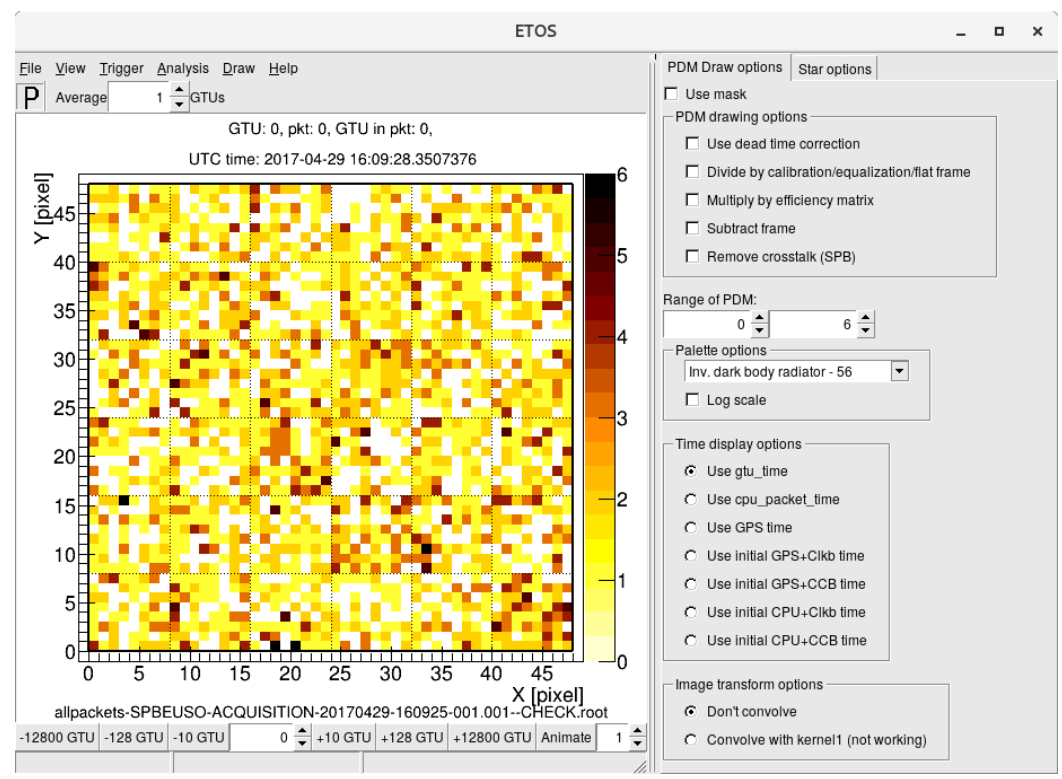

Figure 4: The main window of the "Etos" quick-view programme. Left part shows a single frame with photon counts from the EUSO-SPB experiment [8]. Right part shows multiple display options.

experiment, use of a program in different environments (different distributions of Linux, MacOSX and Windows just to name the most popular ones) is often problematic. We avoided this issue by the use of ROOT graphic user interface elements, which make the program almost platform independent, requiring only a properly configured installation of ROOT and Python.

The main task of the quick-view program is to provide a quick look into the data, displaying the photoelectron counts on the focal surface (fig. 4). The main window allows selecting which GTU's data should be displayed and tracing the changes on the focal surface with time. A specific GTU can be selected, or the GTUs can be changed sequentially manually or as an animation. In addition, averages of specified number of frames can be displayed and navigated instead of a single GTU. The software provides both geometrical and electronics coordinates of the pixels in real-time. The display is the ROOT 2D histogram, therefore all the standard ROOT methods are accessible, making it easy to zoom to a selected part of the focal surface, changing the colour scale of the histogram, fitting a mathematical function, smoothing, etc. The histogram can be saved in both graphical and the framework's format for further analysis with additional tools. Clicking on a pixel brings up that pixel's analysis window, which includes the pixel's light curve (plot of photoelectron counts over time) and the counts histogram.

When working with the calibration data, the main window displays the average photoelectron count for the focal surface pixels for a given threshold. In this case, a specific threshold can be selected, changed by fixed values or animated. Similarly to the behaviour in event analysis mode, clicking on a pixel brings up that pixel's analysis window, this time containing its S-Curve and its derivatives, if requested.

Over the time, "Etos" has been enriched with various more advanced analysis functions, such as basic photometry of objects visible on the frame, or several event search algorithms, dedicated to fast (cosmic-rays) and slow (meteors, satellites) events, which produced important results on 
existing EUSO data. In addition, "Etos" can show data from cameras stored in basic graphics format, synchronised with the UV data through a time-stamp.

\section{Conclusion}

We have described raw data format for the Mini-EUSO mission, which distinctive feature is storing of the photon counts on 3 different time-scales. This raw data after transfer from the ISS to the Earth, either physically or by download, is converted to ROOT TTrees based format compatible with all existing EUSO experiments.

The downloaded data can be viewed in quasi-real time with a dedicated quick-view software - "Etos". The adjustment of this programme to extra data coming from the Mini-EUSO instrument is ongoing, however it has already proved an important tool for analysis of the EUSO family experiments' results.

\section{Acknowledgments}

This work was partially supported by Basic Science Interdisciplinary Research Projects of RIKEN and JSPS KAKENHI Grant (22340063, 23340081, and 24244042), by the Italian Ministry of Foreign Affairs and International Cooperation, by the Italian Space Agency through the ASI INFN agreement n. 2017-8-H.0, by NASA award 11-APRA-0058 in the USA, by the Deutsches Zentrum für Luft- und Raumfahrt, by the French space agency CNES, the Helmholtz Alliance for Astroparticle Physics funded by the Initiative and Networking Fund of the Helmholtz Association (Germany), and by Slovak Academy of Sciences MVTS JEM-EUSO as well as VEGA grant agency project 2/0132/17. Russia is supported by the Russian Foundation for Basic Research Grant No 1629-13065-ofi-m, by ROSCOSMOS, by contract 2016-1-U.0, and the Olle Engkvist Byggmästare Foundation.

\section{References}

[1] J.H. Adams Jr. et al., An evaluation of the exposure in nadir observation of the JEM-EUSO mission, Astroparticle Physics, 44 (2013), 76

[2] M. Ricci, Mini-EUSO: a precursor mission to observe and study Atmosphere and Earth UV emission from the International Space Station, these proceedings

[3] H. Miyamoto et al., Performance of the SPACIROC front-end ASIC for JEM-EUSO, 33rd International Cosmic Ray Conference Proceedings, 103

[4] Xilinx. Zynq-7000 All Programmable SoC, URL: https://www.xilinx. com/products/silicon-devices/soc/zynq-7000.html, 2016

[5] M. Bertaina et al., The Mini-EUSO multilevel trigger algorithm and its performance, these proceedings

[6] F. Capel et al., Mini-EUSO flight software and operations on ISS, these proceedings

[7] CERN ROOT framework, http://root.cern.ch

[8] L. Wiencke, A. Olinto, EUSO-SPB Mission and Science, these proceedings 